CLINICAL STUDY

\title{
Searching for somatic mutations in McCune-Albright syndrome: a comparative study of the peptidic nucleic acid versus the nested PCR method based on 148 DNA samples
}

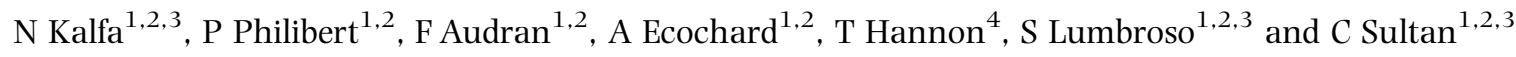 \\ ${ }^{1}$ Unité d'Endocrinologie-Gynécologie Pédiatrique, Service de Pédiatrie 1, Hôpital Arnaud-de-Villeneuve, CHU Montpellier, 34295 Montpellier, France, \\ ${ }^{2}$ Service d'Hormonologie du Développement et de la Reproduction, Hôpital Lapeyronie, CHU Montpellier, 34295 Montpellier, France, ${ }^{3}$ INSERM U540, \\ Hormones et Cancers, Montpellier, France, ${ }^{4}$ Department of Pediatrics, Section of Pediatric Endocrinology, James Withcomb Riley Hospital, Wells Center for \\ Pediatric Research, Indianapolis, Indiana, USA
}

(Correspondence should be addressed to C Sultan; Email: c-sultan@chu-montpellier.fr)

(S Lumbroso is now at Laboratoire de Biochimie, CHU Nimes, France)

\begin{abstract}
Background: Activating mutations of the Gs $\alpha$ gene (GNAS), which encodes for the $\alpha$-subunit of the stimulatory G protein, have been identified in patients with McCune-Albright syndrome (MAS). Accuracy and sensitivity in the molecular diagnosis of MAS is mandatory for optimal therapeutic strategy and adapted follow-up, especially for incomplete clinical forms of MAS. To date, the highly sensitive nested PCR method with intermediary digestion by a restriction enzyme at the mutation site is one of the most widely used techniques. This study evaluated a new diagnostic method using a peptidic nucleic acid (PNA) and compared it with the nested PCR method.

Material and methods: One hundred and forty-eight DNA samples from eighty-eight patients presenting clinical symptoms compatible with MAS were included. The DNA samples were mainly obtained from peripheral blood, ovarian tissue or cyst liquid, and bone lesions. The nested PCR method required 4 days. PNA clamping required 1.5 days and utilized the higher thermal stability and specificity of PNA-DNA coupling to inhibit PCR product formation. Direct sequencing was subsequently performed in all cases.

Results: The sensitivity of mutation detection was $54 \%(n=80)$ for nested PCR and $46.6 \%(n=69)$ for PNA $(P>0.05)$. The 11 cases where PNA failed to detect the mutation were mainly incomplete and atypical clinical forms of MAS $(n=10 / 11)$. The cost per sample was 50 euros for PNA clamping versus 136 euros for nested PCR.

Conclusion: PNA clamping is a rapid, reliable, and economical method to diagnose MAS. It should be the first-line diagnostic method, although negative results, especially for incomplete clinical forms of MAS, should be confirmed by nested PCR.
\end{abstract}

European Journal of Endocrinology 155 839-843

\section{Introduction}

The McCune-Albright syndrome (MAS) is characterized by a triad of physical signs: café-au-lait pigmented skin lesions, polyostotic fibrous dysplasia (FD), and endocrine dysfunction, which particularly manifests as peripheral precocious puberty in girls $(1,2)$. Other hyperfunctional endocrinopathies have been reported such as pituitary adenomas secreting growth hormone and/or prolactin (3), hyperthyroidism (4), autonomous adrenal hyperplasia (5), and hypophosphatemic osteomalacia (6). The endocrine glands that are hyperactive in MAS have in common a response to extracellular signals by the adenylate cyclase (AC)-cAMP pathway and constitutionally elevated AC activity (7). Activating mutations in the guanine-nucleotide binding protein
(G-protein) $\alpha$-subunit $(\mathrm{Gs} \alpha)$ that stimulates AC have been confirmed as the molecular cause of MAS (8). The mutation is nearly always a substitution of the residue arginine at position 201 by histidine or cysteine. The sporadic occurrence of the syndrome, the variable involvement of endocrine glands, and the characteristic pattern of skin and bone lesions, which follows lines of embryologic development, are all in accordance with the mosaic distribution of abnormal cells due to the post-zygotic occurrence of the GNAS mutation (9).

Given these features, it has become apparent that MAS is an extremely heterogeneous disease and that a number of atypical and partial forms exist beyond the classic MAS. It has been hypothesized that identification of GNAS-activating mutations may lead to earlier diagnosis in patients with partial forms of MAS, thereby 
improving management of this diffuse and potentially severe endocrinopathy (10-12). This is only realistic if an accurate, sensitive assay for mutation detection is available. Since GNAS-activating mutations occur in a mosaic distribution, a method that can detect even a low number of mutant alleles is required. Two main methods based on a PCR have been reported: the use of a restriction enzyme with nested PCR to selectively digest the wild-type allele and a peptidic nucleic acid (PNA) primer to inhibit amplification of the wild-type allele (12-14) with or without mutant ratio determination (15).

The basis for choosing between these two techniques has not been clearly established, however, and to date no systematic comparisons in a large series of patients have been carried out. The aim of this study was to evaluate and compare the reliability of these two methods in the molecular diagnosis of patients suspected of having MAS and thus to propose a rationalized use of these techniques.

\section{Patients and methods}

\section{Patients}

Through a European collaborative project, 88 children presenting with one or several signs of MAS were included. Informed consent was obtained from all patients' parents in accordance with institutional guidelines. Eighty percent were girls $(n=70)$. Classic presentations with the triad of precocious puberty, skin lesions, and osteotic FD were seen in $26 \%$ of the cases $(n=23)$. One-third of the children $(n=29)$ showed two of the three signs, and $41 \%(n=36)$ had only one sign at the time of diagnosis, mainly precocious puberty. All of these 36 patients had consulted for a premature thelarche, accelerated growth velocity and 21 of them had recurring ovarian cysts.

\section{DNAs}

The number of DNAs studied $(n=148)$ was greater than the number of patients $(n=88)$ because different tissue samples were taken from the same patient in several cases. DNA was thus extracted from various tissues. Peripheral blood leukocytes (PBL) were the most frequently studied specimen $(n=71)$. The ovary, with ovarian tissue $(n=33)$ or cystic fluid $(n=16)$ obtained from cystectomy or ovarian puncture, was studied in 49 cases. Bone samples could be studied in 15 cases. More rarely, we were able to extract DNA from the adrenal glands $(n=5)$, the liver $(n=4)$, and the endometrium $(n=4)$.

\section{DNA extraction}

DNA was obtained from PBL in EDTA-containing tubes using the QIAamp DNA Blood Kit (Qiagen). The tissue biopsies, whether fresh, frozen, or preserved in paraffin, were submitted to proteinase $\mathrm{K}$ treatment before extraction. Moreover, pathologic zones on the slide were first identified to increase the sensitivity of detection by limiting the search to abnormal cells.

\section{Identification of the Arg 201 mutation by nested PCR and restriction endonuclease digestion}

We used a modification of the method reported by Candeliere et al. (13) that enables selective enrichment of mutated DNA. The principle is based on the use of a modified primer to obtain a PCR product from normal DNA that can be digested by a restriction enzyme (EagI), whereas the PCR product obtained from mutated DNA is resistant to this enzyme. PCR amplification to introduce a site-directed mutation was performed first (sense primer: TTTTGTTTCAGGACCTGCTTCGCGGC, antisense primer: AAGCGTTCTTTACG AACAGCCĀAGC). After the sitedirected mutagenesis step, the PCR product was treated with EagI (New England Biolabs, Beverly, MA, USA) prior to further amplification. Digestion was carried out in a $10 \mu \mathrm{l}$ reaction composed of $1 \mu \mathrm{l}$ NEB3 $10 \times$ buffer, $5 \mu \mathrm{l}$ PCR product, $0.5 \mu \mathrm{l}$ endonuclease $(50 \mathrm{U} / \mu \mathrm{l})$, and $3.5 \mu \mathrm{l}$ water at $37{ }^{\circ} \mathrm{C}$ overnight. For use in subsequent amplifications, the digested PCR product was diluted in the ratio of 1:50 with water. Successive PCR and enzyme digestion steps result in an enrichment of the mutated allele. The sense and antisense primers were those described by Candeliere et al. (13) (second step antisense primer ACAGCCAAGCCCACAGCATCCTAG, third antisense primer: GGTTATTCCAGAGG GACTGGGGTGAA). Amplifications were performed with Qiagen DNA Polymerase in a final volume of $50 \mu \mathrm{l}$ using standard conditions. Before enzymatic digestion, the PCR product was purified using QIAquick PCR columns (Qiagen).

Two successive steps of PCR and digestion were used in this study to limit the risk of contamination due to the nested PCR. After purification, the final PCR products were sequenced with the internal antisense primer.

\section{Identification of the Arg201 mutation with PNA primer}

A technique with a PNA primer to inhibit the amplification of the wild-type allele has been described $(10,12$, 14). PNAs form hybrids with DNA that are stable and more sensitive to internal base mismatches with their DNA complement. The PNA primer that is complementary to the wild-type sequence overlaps the binding site of the sense PCR primer and prevents amplification of the wild-type allele. The PNA primer will not bind to the mutant sequence and the mutant allele is thus amplified during PCR. The sequences of the PNA and primers were as follows: PNA (Applied Biosystems, Courtaboeuf, France): Gly-NH2-CGCTGCCGTGTC-HAc; sense primer: 5'-GTTTCAGGACCTGCTTCGC-3'; and antisense primer: 5'-GCAAAGCCAAGAGCGTGAG-3'. The PCRs contained 
200-500 ng genomic DNA, 2.5 U Qiagen DNA Polymerase, $1 \mu \mathrm{l}$ each of sense and antisense primer, and $2 \mu \mathrm{l}$ PNA, in a final volume of $50 \mu \mathrm{l}$. The PCR conditions were as follows: denaturation at $94{ }^{\circ} \mathrm{C}$ for $15 \mathrm{~min} ; 40$ cycles at $94{ }^{\circ} \mathrm{C}$ for $30 \mathrm{~s}$ (denaturation), $68{ }^{\circ} \mathrm{C}$ for $60 \mathrm{~s}$ (hybridization), $55{ }^{\circ} \mathrm{C}$ for $30 \mathrm{~s}$ (annealing), and $72{ }^{\circ} \mathrm{C}$ for $60 \mathrm{~s}$ (extension) with a final extension at $72{ }^{\circ} \mathrm{C}$ for $7 \mathrm{~min}$ (14). The PCR products were analyzed by gel electrophoresis and sequenced by PCR with the use of an internal primer (5'-GGTTATTCCAGAGGGACTGGGGTGAA-3').

In all the cases and for both methods, negative controls with normal DNA and no DNA were simultaneously run to ensure the absence of contamination. Sequencing reactions were repeated twice with at least two different PCR products for each method.

\section{Statistical analysis}

Statistical analysis was performed with the SPSS 11.1 program. $\chi^{2}$-tests for qualitative data and Student's $t$ tests for quantitative data were used. Significance was accepted at $P<0.05$.

\section{Results}

All results are summarized in Table 1 . The nested PCR with restriction endonuclease digestion detected an activating mutation of GNAS in $54 \%(n=80)$ of the samples. The sensitivity of the PNA method was $46.6 \%$. The sensitivity of PNA was elevated for DNA extracted from ovary (ovarian tissue, $66.7 \%$ or cyst liquid, $81.2 \%$ ) and from bone $(80 \%)$, and lower from DNA extracted from blood leukocytes (36.6\%). In cases of positive PNA clamping from blood leukocytes DNA in a patient, the mutation was always detected on the ovarian or bone tissue. The difference between the sensitivity of mutation detection of these two methods was not statistically significant. However, in 11 cases the PNA method did not detect the mutation detected by nested PCR. Most of the samples in which this discrepancy occurred were DNAs from patients with an incomplete clinical form of MAS or an atypical form $(n=10 / 11)$. Seven cases were incomplete forms (five isolated precocious pseudopuberties and two isolated FDs) and three had atypical presenting symptoms (one neonatal cholestasis and two adrenal hypercorticisms). The average cost per sample and the wait-time for results using the nested PCR were more than twice those of PNA clamping.

\section{Discussion}

Confirming a clinical diagnosis of MAS with the complete triad of signs is of academic interest but has little impact on patient management. In contrast, incomplete or atypical clinical forms of MAS are often quite difficult to recognize, and rapid and reliable molecular methods would be useful for early diagnosis and adapted therapeutic management of these patients. Evidence of a GNAS mutation in a child presenting incomplete or atypical signs is sufficient for diagnosis and the immediate implementation of a strategy of active surveillance so that other signs of MAS (precocious puberty, FD) can be quickly met with adapted management.

The post-zygotic occurrence of the GNAS mutation leading to MAS is responsible for the mosaic distribution of mutant-bearing cells. The subsequent number of affected cells in a tissue may be very low and this complicates the identification of mutant GNAS. The molecular diagnosis of MAS is therefore highly dependent on the sensitivity of the detection method. One of the most widely used methods is a highly sensitive PCRbased technique that allows the selective enrichment of mutated DNA (13). In contrast to the several steps described by Candeliere et al. (13), we recommend only two steps of digestion and several negative controls to limit the risk of contamination inherent to nested PCR. However, this technique is not easily transposed for all mutation sites and has been shown to have variable sensitivity. One of the most recent and promising

Table 1 Comparison between nested PCR with restriction endonuclease digestion and PNA clamping for the detection of an activating mutation of GNAS in position Arg 201.

\begin{tabular}{lcc}
\hline & PNA clamping & $\begin{array}{c}\text { Nested PCR with restriction } \\
\text { endonuclease digestion }\end{array}$ \\
\hline Overall sensitivity of detection $(n=148)$ & $46.6 \%(n=69)$ & $54 \%(n=80)$ \\
Peripheral blood leukocytes $(n=71)$ & $35.2 \%(n=25)$ & $36.6 \%(n=26)$ \\
Ovary $(n=33)$ & $63.6 \%(n=21)$ & $66.7 \%(n=22)$ \\
Ovarian cyst liquid $(n=16)$ & $43.7 \%(n=7)$ & $81.2 \%(n=13)$ \\
Bone $(n=15)$ & $73.3 \%(n=11)$ & $80 \%(n=12)$ \\
Adrenal gland $(n=5)$ & 0 & $40 \%(n=2)$ \\
Liver $(n=4)$ & $25 \%(n=1)$ & $50 \%(n=2)$ \\
Endometrium $(n=4)$ & $75 \%(n=3)$ & $75 \%(n=3)$ \\
Time to results after DNA extraction & 1.5 days & 4 days \\
Average cost per sample & 50 euros & 136 euros \\
\hline
\end{tabular}

No differences reached statistical significance.

ancluding investment costs, operating expenses, and staff salaries. 
methods is based on the use of a PNA primer to block amplification of the normal allele (14). PNAs are synthetic DNA analogs in which the phosphodiester backbone is replaced by repetitive units of $N$ (2-aminoethyl) glycine, to which the purine and pyrimidine bases are attached via a methyl carbonyl linker (16). PNA is a potent DNA mimic in terms of sequence-specific hybridization, and the obtained results show that at physiologic ionic strength PNA/DNA duplexes are generally $1{ }^{\circ} \mathrm{C}$ per base more thermally stable than the corresponding DNA/DNA duplexes (17, 18). Thus, a PNA/DNA complex can effectively block the formation of a PCR product when the PNA is targeted against one of the PCR primer sites. This blockage, termed PNA-directed PCR clamping, allows selective amplification/suppression of target sequences that differ by only $1 \mathrm{bp}$ (19). To date, this method has not been compared with the nested PCR method.

In this study, we show that PNA clamping is a valuable tool for detecting the Arg201 mutation in the molecular diagnosis of MAS. PNA has the advantages of reduced cost and rapid and reliable results. From a technical point of view, PNA may have the added advantage of a limited risk of contamination since only one PCR is applied. Even when the nested PCR method is limited to only two PCRs and digestions and systematically includes negative controls, the risk of contamination should not be underestimated. Nevertheless, we found that PNA clamping might not be as sensitive as nested PCR. This difference did not reach significance but it should be underlined that nested PCR detected an Arg201 mutation in 11 patients who had normal PNA clamping results. An incomplete blockage of the normal DNA amplification by the PNA (19) could explain these false negatives. This result raises two points of interest. First, this finding is not in accordance with those of the previous literature, even though we used the same protocol and primers. Lietman et al. (12) reported that PNA clamping detected the Arg201 mutation in 14 out of 16 patients' blood in a study of 13 patients with a clinical diagnosis of MAS and 3 patients with isolated fibrous bone dysplasia. The authors concluded that PNA clamping provided an enhancement over standard PCR but did not further compare it with the nested PCR. Hannon et al. (10) reported results closer to ours, with the sensitivity of PNA clamping less than 50\% for DNA from blood leukocytes. The authors did not find that PCR was more efficient but the size of their series was too limited $(n=9)$ to provide a significant comparison of the two techniques. The variable clinical criteria of the patient populations (complete triad or atypical MAS) may explain the discrepancies between these studies and ours. Indeed, the finding of a mutation is correlated with the number of clinical signs of MAS (11).

The second interest of our findings is that they lend support to a rationalized cost-effective protocol to screen the Arg201 mutation in patients suspected of having MAS. Our results suggest that PNA clamping could be used as the first-line choice for DNA extracted from tissue and from patients' blood when the complete clinical triad of MAS signs is seen. In these situations, the rate of potentially mutation-bearing cells may be high enough to be reliably detected by this method. Nested PCR could be reserved only for DNA extracted from lowmutated cell content in cases of low clinical suspicion of MAS and for confirmation of negative results of PNA clamping. In the present study, application of this protocol would have saved 2838 euros.

Overall, PNA clamping is a reliable, rapid, and costeffective method for the molecular diagnosis of MAS. Nested PCR nevertheless remains the most sensitive technique, especially for the incomplete and atypical forms of MAS.

\section{Acknowledgements}

This work was supported by a national grant from the Association de Recherche contre le Cancer (ARC: JR/MLD/MDV-P05/5). It will be presented as an oral communication at the next ESPE meeting (Rotterdam, June 2006). We would like to thank O Pescovitz (Department of Pediatrics, Section of Pediatric Endocrinology, James Withcomb Riley Hospital, Wells Center for Pediatric Research, Indianapolis, Indiana, USA) for her technical assistance and review of the manuscript. We would like to thank the following doctors and professors for having sent us MAS patients or the DNA from these patients: Alos (Narbonne), Attouche (Toulouse), Baechler-Sadoul (Nice), Barat (Bordeaux), Baron (Nantes), Bercovici (Brest), Bertrand (Besançon), Bost (Grenoble), Brauner (Paris), Bremond (Marseille), Carel (Paris), Cartigny (Lille), Charbonnel (Nantes), Chaussain (Paris), Colle (Bordeaux), Copelli (BuenosAires), Coutant (Angers), Czernichow (Paris), de Kerdanet (Rennes), de Muinck-Keizer (Rotterdam), Despert (Tours), Drop (Rotterdam), Dumas (Montpellier), Eiholzer (Zurich), Fauser (Rotterdam), Fellman (Besançon), Feron (Orleans), Garandeau (Montpellier), Gillerot (Brussels), Gottrand (Lille), Jouk (Saint-Etienne), Hoorweg-Nijman (Amsterdam), Kacem (Monastir), L'Allemand (Zurich), Lauras (Saint-Etienne), Laven (Rotterdam), Lebouc (Paris), Leger (Paris), Leheup (Nancy), Limal (Angers), Mallet (Rouen), Malpuech (Clermont-Ferrand), Metz (Brest), Nemeth (Stockholm), Nicolino (Lyon), Nivot (Caen), Peterkova (Moscow), Petrus (Tarbes), Pienkowski (Toulouse), Pinto (Paris), Plauchu (Lyon), Puel (Bordeaux), Razafimahefa (Toulon), Richard (Lyon), Rifai (Lille), Semicheva (Moscow), Silva (Porto), Sokal (Brussels), Soskin (Strasbourg), Starzyk (Krakaw), Szarras-Czapnik (Warsaw), Tauber (Toulouse), Thibaud (Paris), Toublanc (Paris), Troller (La Rochelle), Tsaregorotsev (Moscow), Van den Ouweland (Rotterdam), and Weill (Lille). 


\section{References}

1 McCune D. Osteitis fibrosa cystica. The case of a nine year old girl who also exhibits precocious puberty, multiple pigmentation of the skin and hyperthyroidism. American Journal of Diseases and Children $193652743-744$.

2 Albright F, Butler A, Hampton A \& Smith P. Syndrome characterized by osteitis fibrosa disseminata, areas of pigmentation and endocrine dysfunction, with precocious puberty in females: report of five cases. New England Journal of Medicine 1937216 727-746.

3 Cuttler L, Jackson JA, Saeed uz-Zafar M, Levitsky LL, Mellinger RC \& Frohman LA. Hypersecretion of growth hormone and prolactin in McCune-Albright syndrome. Journal of Clinical Endocrinology and Metabolism $1989 \mathbf{6 8} 1148-1154$.

4 Feuillan PP, Shawker T, Rose SR, Jones J, Jeevanram RK \& Nisula BC. Thyroid abnormalities in the McCune-Albright syndrome: ultrasonography and hormonal studies. Journal of Clinical Endocrinology and Metabolism 199071 1596-1601.

5 Mauras N \& Blizzard RM. The McCune-Albright syndrome. Acta Endocrinologica. Supplementum (Copenh) 1986279 207-217.

6 Lee PA, Van Dop C \& Migeon CJ. McCune-Albright syndrome. Long-term follow-up. JAMA 1986256 2980-2984.

7 Vallar L, Spada A \& Giannattasio G. Altered Gs and adenylate cyclase activity in human GH-secreting pituitary adenomas. Nature $1987330566-568$.

8 Weinstein LS, Shenker A, Gejman PV, Merino MJ, Friedman E \& Spiegel AM. Activating mutations of the stimulatory G protein in the McCune-Albright syndrome. New England Journal of Medicine 1991325 1688-1695.

9 Happle R. The McCune-Albright syndrome: a lethal gene surviving by mosaicism. Clinical Genetics 198629 321-324.

10 Hannon TS, Noonan K, Steinmetz R, Eugster EA, Levine MA \& Pescovitz OH. Is McCune-Albright syndrome overlooked in subjects with fibrous dysplasia of bone? Journal of Pediatric 2003 142 532-538.

11 Lumbroso S, Paris F \& Sultan C. Activating Gsalpha mutations: analysis of 113 patients with signs of McCune-Albright syndrome - a European collaborative study. Journal of Clinical Endocrinology and Metabolism 200489 2107-2113.
12 Lietman SA, Ding C \& Levine MA. A highly sensitive polymerase chain reaction method detects activating mutations of the GNAS gene in peripheral blood cells in McCune-Albright syndrome or isolated fibrous dysplasia. Journal of Bone and Joint Surgery. American Volume 200587 2489-2494.

13 Candeliere GA, Roughley PJ \& Glorieux FH. Polymerase chain reaction-based technique for the selective enrichment and analysis of mosaic arg201 mutations in $\mathrm{G}$ alpha s from patients with fibrous dysplasia of bone. Bone 199721 201-206.

14 Bianco P, Riminucci M, Majolagbe A, Kuznetsov SA, Collins MT, Mankani MH, Corsi A, Bone HG, Wientroub S, Spiegel AM, Fisher LW \& Robey PG. Mutations of the GNAS1 gene, stromal cell dysfunction, and osteomalacic changes in non-McCune-Albright fibrous dysplasia of bone. Journal of Bone and Mineral Research 200015 120-128.

15 Karadag A, Riminucci M, Bianco P, Cherman N, Kuznetsov SA, Nguyen N, Collins MT, Robey PG \& Fisher LW. A novel technique based on a PNA hybridization probe and FRET principle for quantification of mutant genotype in fibrous dysplasia/McCuneAlbright syndrome. Nucleic Acids Research 200432 e63.

16 Paulasova P \& Pellestor F. The peptide nucleic acids (PNAs): a new generation of probes for genetic and cytogenetic analyses. Annales de Genetique 200447 349-358.

17 Nielsen PE, Egholm M, Berg RH \& Buchardt O. Sequence-selective recognition of DNA by strand displacement with a thyminesubstituted polyamide. Science 1991254 1497-1500.

18 Egholm M, Buchardt O, Christensen L, Behrens C, Freier SM, Driver DA, Berg RH, Kim SK, Norden B \& Nielsen PE. PNA hybridizes to complementary oligonucleotides obeying the Watson-Crick hydrogen-bonding rules. Nature $1993 \mathbf{3 6 5}$ 566-568.

19 Orum H, Nielsen PE, Egholm M, Berg RH, Buchardt O \& Stanley C. Single base pair mutation analysis by PNA directed PCR clamping. Nucleic Acids Reserach $1993215332-5336$.

Received 28 June 2006

Accepted 21 September 2006 